\title{
Concentration Effects on the Dynamics of Liquid Crystalline Self-Assembly: Time-Resolved X-ray Scattering Studies
}

\author{
Marcel Petri, ${ }^{\dagger}$ Andreas Menzel, ${ }^{\ddagger}$ Oliver Bunk, ${ }^{\ddagger}$ Gerhard Busse, $^{\dagger}$ and Simone Techert $^{*,+}$ \\ ${ }^{\dagger}$ Department of Structural Dynamics of (Bio)chemical Systems, Max Planck Institute for Biophysical Chemistry, \\ 37070 Göttingen, Germany \\ ${ }^{\ddagger}$ Paul Scherrer Institute, 5232 Villigen PSI, Switzerland
}

Supporting Information

ABSTRACT: A manifold of ordering transitions relevant to chemical and biological systems occur at interfaces from liquids to self-assembled soft solids like membranes or liquid crystals. In the present case, we were interested in understanding the phase transition from the microemulsion phase to the liquid crystal phase in terms of their driving forces, i.e., activation energy and entropy. The purpose of this work was to clarify the influence of concentration effects of the amphiphilic molecules on the nature of these self-assembly processes. By photosensitization of the model system (polyalkylglycolether $\left(\mathrm{C}_{10} \mathrm{E}_{4}\right)$, water, decane, and cyclohexane) with laser dyes, we could effectively induce and control the phase transition through the absorption of optical photons. The photo transformation conditions were chosen in such a way that the system was in thermal equilibrium. By

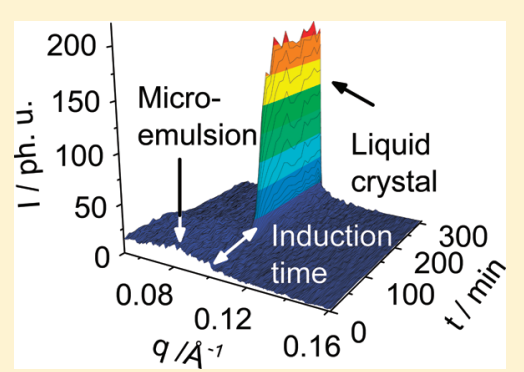
application of time-resolved photo small-angle X-ray scattering we could monitor the conversion process and demonstrate that the surfactant concentration has a direct impact on the activation energy, which is observable through the length of the induction time.

\section{INTRODUCTION}

Ternary liquid crystal (LC) phases are well-known for their huge variety of ordering transitions at the interface between LCs and aqueous phases embracing amphiphilic molecules, polymers, and proteins. ${ }^{1}$ This interface is subjected to large-scale fluctuations arising from self-assembly processes of surfactants in the bulk for particularly at the phase transition from LCs to aqueous phases. For this reason, it is an important task and great challenge to develop effective and environmentally benign methods, which allows rationalization and controlled phase transitions. ${ }^{2}$ In principle, the phase transitions can be either temperature- or laser-induced. Laser-induced self-assembly has been applied to amphiphilic molecules on the gas-liquid interface, ${ }^{3}$ to organic acids at the liquid-solid interface, ${ }^{3}$ and to the liquid-liquid interface. ${ }^{4}$ Absorption of optical photons provides the means to introduce and to control phase changes via light irradiation. A prerequisite limiting these methods to certain special cases is the optical activity of the system under investigation. Then coherent excitation of matter, in order to avoid surface effects, can induce macroscopic changes in the state of the system. So far, the investigated phase transitions were either driven by laser heating or by intermediate formation following optical selection rules. ${ }^{5}$ Photosensitization by means of dye systems extends the possibility of photo inducing phase transitions to any arbitrarily chosen matter, which might be inherently inert against optical excitation. Furthermore, photosensitization allows the initiation of phase transitions, which are driven by Stokes or anti-Stokes optical excitation resulting in a heating or cooling of the surroundings of the dye molecules. Collisional redistribution of radiation or anti-Stokes excitation of a dye ${ }^{6}$ are methods employing laser-irradiation in order to reduce the inner energy of the system. We have introduced this concept showing that ternary LC systems near thermal equilibrium could be easily transformed from a microemulsion (ME) state to the liquid crystalline state. ${ }^{6}$ In a subsequent study, the effect of chain length increase of the hydrophilic molecule tail on the dynamics could be elucidated. A longer chain length accounted for an extended growth rate, which was clarified in terms of activation energy and entropy. ${ }^{7}$ In the present study, we will demonstrate how to switch an isotropically oriented solution to the liquid crystalline state and investigate the influence of concentration effects on the dynamics of such self-assembly processes.

The phase transitions under study are from the quasiternary system $\mathrm{C}_{10} \mathrm{E}_{4}$, water, $n$-decane, and cyclohexane. Variation of the surfactant mass fraction at equal volume fractions of water and oil lead to the phase diagram depicted in Figure 1. Cyan represents an area where two phases coexist, dark gray the ME phase, and white the LC phase. The dots represent points of the phase boundaries at specific concentrations $c$ and temperatures $T$ as measured by the polarized screening technique. The arrows indicate points in the phase diagram for which the ME/LC phase transition has been photoinduced. The structures of the microemulsion as well as LC phase are depicted in a schematic manner in the inset. Their characteristic structural properties, coherence length, and periodicity will be explained in the following. In the two-phase region, the surfactant-rich oil phase

\footnotetext{
Received: November 12, 2010

Revised: February 3, 2011

Published: February 25, 2011
} 


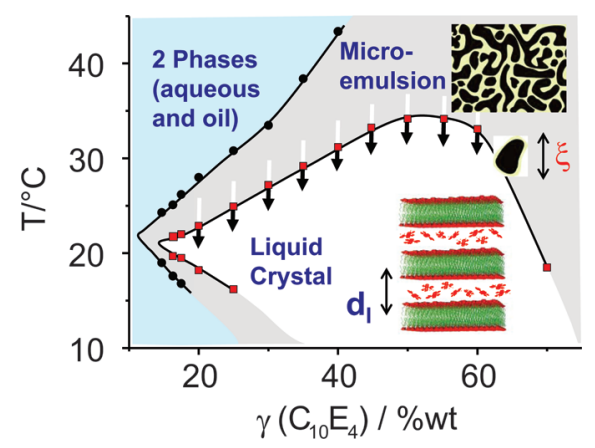

Figure 1. Phase diagram of the system $\mathrm{C}_{10} \mathrm{E}_{4}$, water, $n$-decane, and cyclohexane at equal volume fractions of water and oil. Cyan represents an area where two phases coexist, dark gray the ME phase, and white the LC phase. The dots represent points of the phase boundaries as been measured by the polarized screening technique. Arrows indicate the concentrations at which the phase-transition has been photoinduced from the microemulsion to the LC phase. Inset: schematical drawings of the corresponding phases with the assignment of characteristic length scales like correlation length $\xi(\mathrm{ME})$ and distance $d$ between the lamellae (LC).

is in equilibrium with an aqueous oil poor phase. ${ }^{8}$ Temperature changes the affinity of the amphiphilic molecules for the aqueous and oil phases for a system based on nonionic surfactants. In the microemulsion phase, the amphiphilic solutions appear macroscopically as one phase. In reality, the phase is separated on the microscopic scale with the oil and water domains held in contact by the surfactant molecules. At concentrations where the microemulsion contains equal volumes of water and oil, a bicontinuous microemulsion consisting of interpenetrating domains with equal length scales is formed. ${ }^{9}$

At intermediate temperatures the formation of lyotropic LCs with a long-range lamellar order and anisotropic behavior of hydration water is favored. ${ }^{10}$ Interestingly, the macroscopic appearance of the LC formed after optical excitation of the solution is quite distinct from the one formed after temperature reduction. Concomitantly to laser cooling, the laser field interacts through the induced dipole moment of the molecule finally resulting in preferred orientation. As a consequence of this interaction, the resulting LC exhibits a unique texture ("oily streaks") over the whole volume of the capillary.

A temperature-induced phase transition, in contrast, leads to the creation of isotropically distributed liquid crystalline domains. Figure 2a shows a characteristic micrograph of such a dyedoped microemulsion as the initial state. The differences of a LC phase created by photo induction and a temperature-induced phase transition are well visible in parts B and C of Figure 2. The first displays long stripes along the direction of the capillary whereas the latter (temperature-induced LC phase) shows a mosaiclike pattern due to a random arrangement of the liquid crystalline domains. All the photographs were taken with crossed polarizers $20 \mathrm{~h}$ after the phase transition had been induced. Figure 2 emphasizes the possibility to photo induce self-assembly and furthermore to control the pattern formation as a result of the self-assembly process. In the following, we will therefore investigate the nature and the mechanism of this self-assembly process which leads to such pattern structures in the LC phases. We present our studies on the kinetic and structural origin of such pattern formations, carried out by means of time-resolved $\mathrm{X}$-ray scattering. In the following, we will give an overview about the preparation details and experimental procedure.

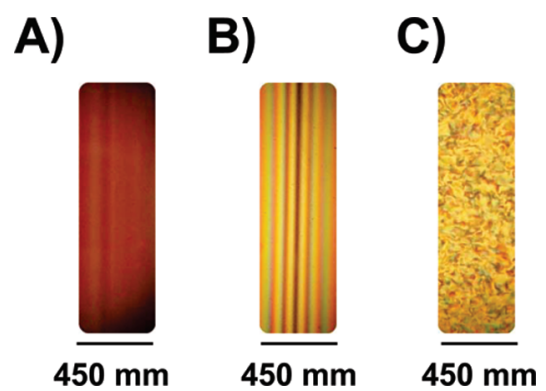

Figure 2. (A) Micrograph of the initial dye doped microemulsion. (B) Polarized light micrographs of the lamellar phase after photo induction. (C) Polarized light micrographs of the lamellar phase after temperatureinduced phase transitions.

\section{EXPERIMENTAL SECTION}

2.1. Preparation. The systems were prepared as follows: Tetraethyleneglycolmonodecylether $\left(\mathrm{C}_{10} \mathrm{E}_{4}\right)$ and $n$-decane both with $98 \%$ purity were purchased from Fluka, and no further purification was done. The surfactant agent was dissolved in a ternary solution at various concentrations. The first volume fraction at $\alpha$, defined as $\alpha=V_{\text {oil }} /\left(V_{\text {oil }}+V_{\text {water }}\right)$, was maintained at $\alpha=50 \%$. The range of surfactant concentration at which the phase transition was photoinduced was chosen to be close to room temperature $\left(24-25{ }^{\circ} \mathrm{C}\right)$. The solutions were prepared from bidistilled water (polar solvent), $n$-decane, or octane ( $98 \%$ purity, Fluka) (99\% purity, Fluka) and cyclohexane (99\% purity, Merck). The dye Rh101, (99\%, $c=2 \times 10^{-5}$ M, Lambda Physics) was added to the mixtures without further purification. The mixtures were stirred at room temperature for $1 \mathrm{~h}$ in a thermostat. Further preparation details are described in Petri et al. ${ }^{7}$

2.2. Methods. Time-resolved small-angle X-ray scattering (TR-SAXS) is one of the most useful techniques to characterize the lamellar structure and to investigate the emergence of crystalline order. In the present experiment, the time-evolution of a Bragg peak has been monitored as a function of concentration with a time resolution of $t=30 \mathrm{~s}$. The SAXS experiments have been performed on a home-based Kratky camera using $\mathrm{Cu}$ $\mathrm{K} \alpha(\lambda=1.542 \AA)$ and additionally at the cSAXS beamline of the Swiss Light Source to which an optical laser $(\lambda=623.8 \mathrm{~nm}, p=3$ $\mathrm{mW})$ was coupled. Details on the experimental setup can be found elsewhere. ${ }^{11}$

\section{RESULTS AND DISCUSSION}

3.1. ME/LC Phase Transition. The following gives a detailed analysis of the structural changes underlying the ME-LC phase transition, which is depicted in Figure 1. Before light illumination, the ternary system was stabilized at higher temperatures. Anti-Stokes excitation of the dye molecules photoinduces the phase transition, which leads to an increase of the long-range order liquid crystalline domains. The time evolution of the LC phase has been followed by investigating its Bragg reflection in the SAXS regime. Upon light illumination, the integral intensity of the microemulsion diffuse scatter decreases meanwhile a LC reflection appears and increases. In a three-dimensional plot, Figure 3 shows the scattering data during the phase-transition process with a surfactant concentration of $\gamma=45 \mathrm{wt} \%$. It is clearly seen that the system undergoes the phase-transition after an induction time of $t=110 \mathrm{~min}$. The induction time is referred to as the time between the beginning of the irradiation of the 


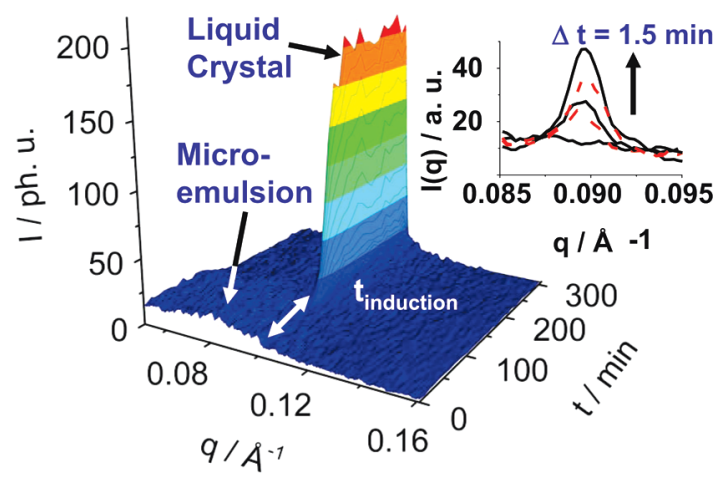

Figure 3. Photoinduced ME-LC phase transition investigated by TRSAXS. The decrease of the ME and the concomitant increase of the Bragg peak, which testifies the emergence of a lamellar structure, are shown. The monoexponential nucleation and growth period is preceded by a concentration dependent induction time. Inset: Traces of the LC scattering pattern right after the lapse of the induction time.

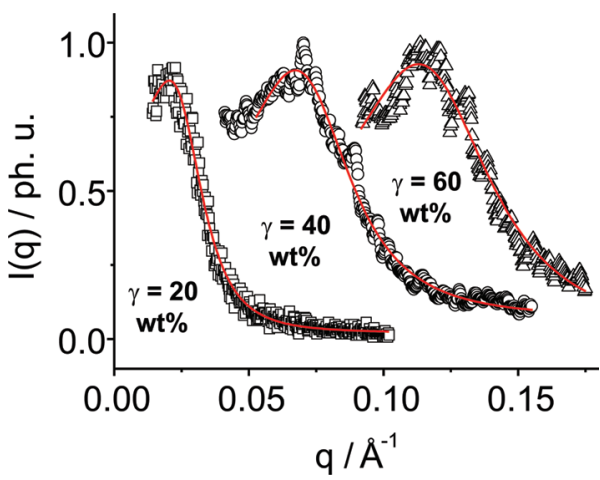

Figure 4. Static SAXS pattern from the pure microemulsion phase for surfactant concentrations of $\gamma=20 \mathrm{wt} \%(\square), \gamma=40 \mathrm{wt} \%(\bigcirc)$, and $\gamma=$ $60 \mathrm{wt} \%(\Delta)$. The microemulsion peaks broaden and move to higher wave vectors $q$ with increasing surfactant concentration. The symbols depict the measured data; the lines are regression fits according to the Teubner-Strey equation.

sample and the appearance of the first detectable scattering signal of the LC Bragg reflection. The phase-transition itself is a much faster process as depicted in the insert of Figure 3. Here it is shown that a considerable increase of the Bragg peak occurs within $1.5 \mathrm{~min}$. Within $t=30 \mathrm{~min}$, the Bragg peak reaches its maximum intensity. Systems that are not dye sensitized neither exhibit major changes with respect to their X-ray intensities nor undergo a phase transition. To understand better the kinetics of the self-assembly and its driving force, we will start with shedding light on the initial and final states. The initial state is characterized as the ME phase, the final state as the LC phase.

3.2.1. Initial State: Structural Properties of the ME. Despite its lack of long-range order, the regular alteration of water and oil domains in the bicontinuous structure of the microemulsion generates a pronounced correlation peak of the scattering intensity at wavevectors $q=2 \pi / d$. Here, $d$ is the average distance between neighboring oil-oil or water-water domains (see Figure 1). Fitting of the experimental data of the initial ME phase with the well-known Teubner-Strey equation yields the characteristic $d$ spacing and the correlation length $\xi$ for the microemulsion. ${ }^{12}$ Regression fits to the experimental SAXS data for the microemulsion phase according to the Teubner-Strey

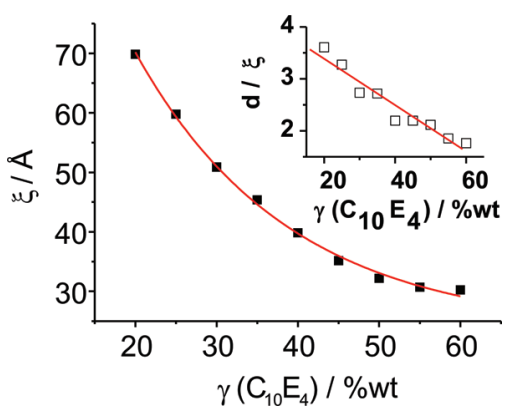

Figure 5. Structural properties of the ME phase. The correlation length $\xi$ characterizes the size of the interpenetrating water and oil domains. It exhibits an exponential decline for increasing surfactant concentrations. Inset: The ratio $d / \xi$ serves as a measure for the degree of ordering in the systems. $d$ is assigned to the interdomain distance. Surfactant-rich microemulsions are more ordered than their surfactant-poor counterparts.

equation are shown in Figure 4. Within the kinematic approach, for every time point $t$ the microemulsion diffuse X-ray scattering signal is defined as

$$
I(t) \propto \frac{1}{a_{2}+c_{1}(t) q^{2}+c_{2}(t) q^{4}}+I_{\mathrm{BKG}}
$$

where $I_{\mathrm{BKG}}=$ background scatter signal, $a_{2}$ is a time-independent scaling factor and $c_{1}(t)$ and $c_{2}(t)$ are the concentrations of the two components oil and water. The photo induction of the phase transition is thermotropic (vertical excitation in the phase diagram Figure 1), resulting in a redistribution of the oil and water components within the bulk. Therefore, $c_{1}=c_{1}(t)$ and $c_{2}=$ $c_{2}(t)$. From eq 1 the correlation length $\xi(t)$ and the dispersion $d(t)$ with the quasiperiodic repeat distance $d(t) / 2 \pi$ can be extracted to

$$
\xi(t)=\left[\frac{1}{2}\left(\frac{a_{2}}{c_{2}(t)}\right)^{1 / 2}+\frac{1}{4} \frac{c_{1}(t)}{c_{2}(t)}\right]^{-1 / 2}
$$

and

$$
d(t)=2 \pi\left[\frac{1}{2}\left(\frac{a_{2}}{c_{2}(t)}\right)^{1 / 2}-\frac{1}{4} \frac{c_{1}(t)}{c_{2}(t)}\right]^{-1 / 2}
$$

The correlation length $\xi$, also visualized in Figure 1, is attributed to the size of the adjacent water and oil domains of the initial $\mathrm{ME}$ at an average interdomain distance $d$. The Teubner-Strey model has been widely used to describe scattering from aqueous MEs stabilized by nonionic surfactants. The ME peaks broaden and move to higher wavevectors $q$ with increasing surfactant concentration. This means that the size $\xi$ and the distance $d$ of the oil and water domains decrease as the surfactant concentration increases. Figure 5 demonstrates the variation of the domain size as a function of surfactant concentration.

For a large number of bicontinuous microemulsions, ratios of $d / \xi$ ranging from $d / \xi=1.7$ to $d / \xi=4$ have been reported. ${ }^{9 \mathrm{a}}$ This ratio indicates the degree of ordering of the oil-water domains induced by the self-assembled amphiphilic monolayers. The smaller the ratio, the more ordered the system. Hence, minimal values are adopted at a temperature where the oil-water interface exhibits a pronounced minimum. Limits of the dispersion of the domain size arise from the folding of the amphiphilic film. 
Very small dispersions of the domain sizes lead to a first-order phase transition to the liquid-crystalline phase due to entropy effects, whereas at large dispersions the fluctuations of the amphiphilic film become so great that the bicontinuous microemulsions break up and form a droplet structure. The $d / \xi$ ratios derived from the experimental data measured at temperatures just above the phase transition temperature exhibit a decrease of $d / \xi$ with increasing amphiphile concentration as displayed in the inset of Figure 5. At constant temperatures, for extreme surfactant concentrations of $\gamma=20$ wt $\%$ and $\gamma=60$ wt $\%, d / \xi=3.61$ and $d / \xi=1.76$ have been determined, respectively. This is an indication that a higher surfactant concentration leads to a more ordered structure. Monte Carlo simulations carried out for surfactant concentrations ranging from $\gamma=20$ wt $\%$ to $\gamma=40$ wt $\%$ in chemically related systems are in accordance with our observations. ${ }^{13}$ With these simulations taken into account, the following picture arises for our data: at low surfactant concentrations (e.g., $\gamma=20$ wt \%), the domain sizes of the oil or water domains within the bicontinuous structure of the ME adopt large values of the correlation length with $\xi=70 \AA$. Concomitantly, the characteristic repeat unit reaches up to $d=250 \AA$ while the length of the amphiphilic molecule is constantly around $l \approx 10 \AA$. This means that the ensemble is dominated by a relatively low number of spacious domains. In contrast, at high surfactant concentrations these huge domains have made space for a large number of small-sized domains characterized by low correlation lengths and repeat distances.

3.2.2. Final State: Structural Properties of the LC. After the photoinduced self-assembly process has come to an end, almost the entire solution is transformed to the LC phase. In the following, we will describe this final phase. To analyze the structure of the liquid crystalline phase the remaining scattering pattern contribution of the ME modeled by the Teubner-Strey equation was subtracted. The isolated Bragg reflection was further fitted within the framework of the Porod equation, a first-order Bessel function. ${ }^{14}$ Their time-dependence is defined as

$$
q^{4} I(q, t) / I^{0} \approx \sin ^{2}(L(t) q / 2) \frac{\sin ^{2}(N(t) d(t) q / 2)}{\sin ^{2}(d(t) q / 2)}
$$

where the parameters are defined as $I^{0}=8 \pi N_{\text {cryst }} A\left(\rho_{\mathrm{m}}-\rho_{\mathrm{p}}\right) d$, $N=$ number of lamellae, $d=$ spacing, $L=$ thickness, $A=$ surface of the lamellae, $N_{\text {cryst }}=$ number of (identical) LCs, $\rho_{\mathrm{m}}=$ electron density of the interlayer, $\rho_{\mathrm{p}}=$ electron density of the lamella layer. On the basis of the assumption that the electron density changes as a function of time can not be resolved by the resolution of the scattering momentum they are kept constant. ${ }^{7}$ Figure 6 presents the experimental X-ray scattering pattern in form of the so-called Porod plot together with the regression fits according to eq 4. Two fundamental trends are observed. First, the sharp LC reflection shifts linearly to higher wavevectors in the reciprocal space from $q=0.0359 \AA$ to $q=0.132 \AA$ for $\gamma=20$ wt $\%$ and $\gamma=60$ wt $\%$ surfactant concentration, respectively. Hence, the interlayer distance between the lamellae decreases with increasing surfactant concentration. Second, the number of lamellae increases monoexponentially as a function of concentration.

The distance and number of the lamellae in the crystallites derived from this data evaluation strategy are summarized in Figure 7. At the top, it is shown that the crystallites of the lamellar phase with high surfactant concentrations consist of more lamellae with smaller interlayer distances than those built from
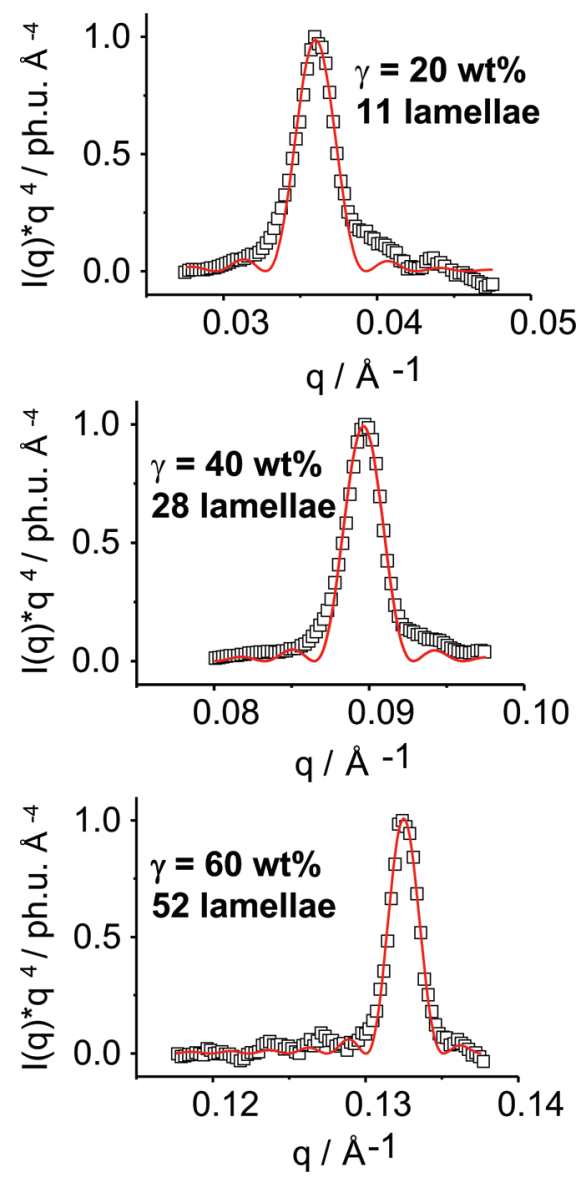

Figure 6. Porod fitting of the $\mathrm{LC}$ reflection allows for the determination of several experimental structural parameters as a function of concentration such as the lamellar periodic unit $d$, the thickness of the lamella $L$, and the number of the lamella $N$. The peaks are shifted to higher wavevectors $q$ with increasing surfactant concentration, i.e., the distance between the lamellae decrease.

solutions with low surfactant concentrations. Our observations of a variation of the peak position as a function of concentration are in good consistence with comparable aqueous lamellar phases of $\mathrm{C}_{12} \mathrm{E}_{5}{ }^{15}$ and $\mathrm{C}_{14} \mathrm{E}_{4}{ }^{16}$ The shift in position has been attributed to a one-dimensional swelling of the lamellae. The effective headgroup area $a_{s}$ occupied by the surfactant is determined according to eq 5

$$
a_{\mathrm{s}}=\frac{M q}{\pi \gamma N_{\mathrm{A}} \rho}
$$

where $M$ is the molecular weight of the LC, $q$ the X-ray scattering wavevector, $\gamma$ the surfactant volume fraction, $N_{\mathrm{A}}$ the Avogardo constant, and $\rho$ the surfactant density of the LC phase. The effective headgroup area $a_{\mathrm{s}}$ of the amphiphilic molecule increases in the range of $\gamma=20 \mathrm{wt} \%\left(a_{s}=33 \AA^{2}\right)$ to $\gamma=40$ wt $\%\left(a_{s}=42 \AA^{2}\right)$ while being constant at higher concentrations as plotted in the bottom of Figure 7 . We propose that the lamellae are more swollen at higher concentrations since the interlayer distance is smaller and the water molecules are tighter bound to the surfactants. At higher concentrations, however, the hydration shell stops growing due to steric reasons. Comparison of our finding to the experimental data of Atkin confirms constancy of the 


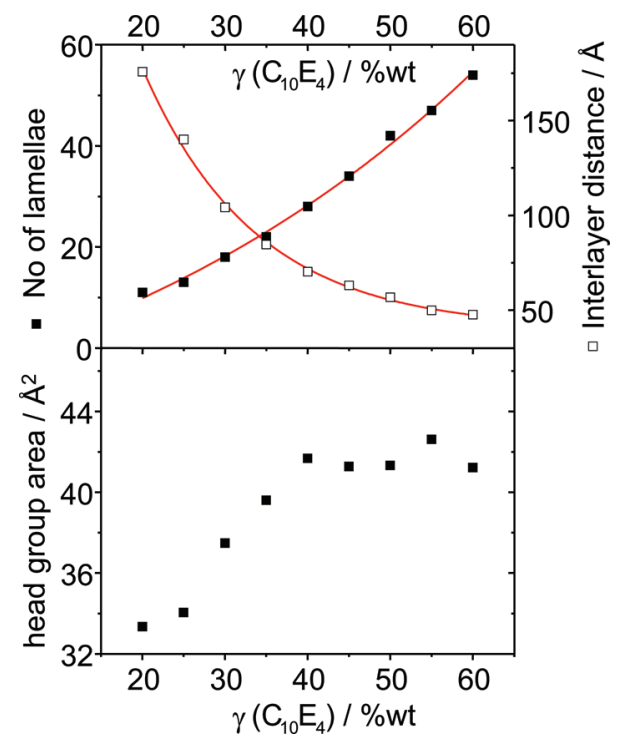

Figure 7. Top: Variation of the number of lamella (left) and their interlayer distance (right) as a function of surfactant concentration. Crystallites of the lamellar phase having high surfactant concentrations consist of a major number of lamellae with a minimal interlayer distance. Bottom: Concentration dependence of the effective headgroup area of the amphiphilic molecules.

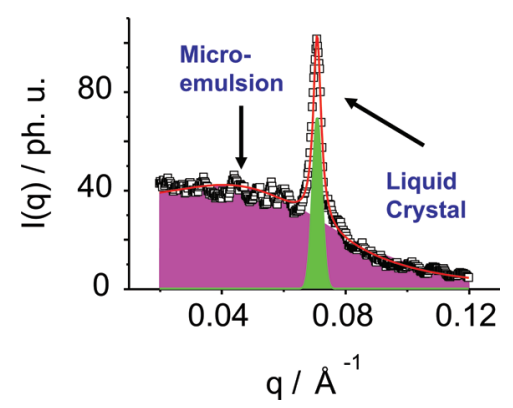

Figure 8. Coexistence of $\mathrm{ME}$ and $\mathrm{LC}$ phase at $t=15 \mathrm{~min}$. The experimental scattering data were fitted by applying a convolution of a Gaussian function (describing the LC diffraction peak) with the Teubner-Stey equation (describing the ME diffuse scatter signal).

headgroup area per molecule in the surfactant range of $\gamma=40$ wt $\%$ to $\gamma=55 \mathrm{wt} \%$. Interestingly, the headgroup area of the aqueous lamellar phase of $\mathrm{C}_{12} \mathrm{E}_{5} \quad a_{\mathrm{s}}=42.9 \AA^{2}{ }^{15-17}$ is as capacious as for $\mathrm{C}_{10} \mathrm{E}_{4}$, indicating that only the first ethoxy units strongly interact with the surrounding water. At low surfactant concentrations, the water molecules assemble loosely around the polar tail of the surfactants until covering the total area.

Besides the investigation of structure, TR-SAXS allows to investigate the dynamics of self-assembled systems and the structural dynamics. After characterizing the initial and end states of the phase transformation, we will now take a closer look on the kinetics underlying the phase transition itself. To obtain a complete picture, the study of the crystallization mechanism covered all relevant time scales of this process. Coming back to Figure 3, for each point in time, the scattering data have been analyzed according to the Teubner-Strey equation (ME signal) and a Gaussian shape (LC signal) regarding the relative contribution of the ME and lamellar phase, respectively. The

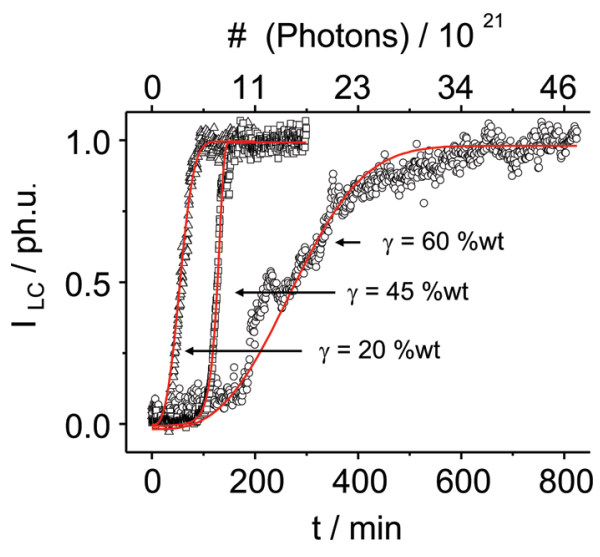

Figure 9. Overlay of the transformation profile showing the concentration dependence of the photoinducedphase transition. After a characteristic induction time, the normalized integral intensity of the LC increases as a function of irradiation. The curves are regression fits of the data according to the homogeneous dispersive kinetics theory allowing the determination of the activation energy $E_{\mathrm{A}}$.

background was determined based on the diffuse scatter in the high $q$ range $(q=0.16 \AA)$ and mathematically treated as a constant. Figure 8 visualizes the coexistence of the ME and LC phase after $15 \mathrm{~min}$ of illumination. The integral intensities which are proportional to the mass fraction of the two phases were normalized for clarity's sake. The diffuse scattering signal arising from the ME phase is depicted in magenta and the LC scattering contribution (Gauss shape) in green.

The integral intensities of the evolving LC scattering signal for the entire conversion process are shown as a function of time on hand of three exemplary surfactant concentrations in Figure 9. This data set serves to demonstrate that an increase in surfactant concentration provokes an extension of the induction period. The representative growth time determined as $\tau_{\mathrm{LC}}=22.4 \mathrm{~min}$ for a surfactant concentration of $\gamma=20 \mathrm{wt} \%$, follows a first-order kinetic law. The fits of this simplified model are given in the Supporting Information. The characteristic time constants for the exponential growth $\tau_{\mathrm{LC}}=22$ $\min (\gamma=20 \mathrm{wt} \%), \tau_{\mathrm{LC}}=15 \mathrm{~min}(\gamma=45 \mathrm{wt} \%)$, and $\tau_{\mathrm{LC}}=165$ $\min (\gamma=60 \mathrm{wt} \%)$ do not feature a trend that is correlated to the surfactant concentration.

From Figures 3 and 9 it is clear that the monoexponential nucleation and growth period is preceded by a pronounced induction time which increases with increasing surfactant concentration. Commonly, the induction time is referred to as the time between the beginning of the irradiation of the sample and the appearance of the first detectable scattering signal of the very first clusters of supernucleus size. The formation of nuclei is essentially a dynamic process dominated by the competition between the reduced bulk free energy and the increased surface free energy. ${ }^{18}$ Thus, the state of metastability during which long-range density fluctuations occur is directly represented by the length of the induction time with long-range density fluctuations being the basic principle of the occurring ordering phenomena. The close correlation between surfactant concentration and length of the induction time is pointed out in Figure 10.

3.3. Dispersive Kinetics. A complete analysis of the transformation curves beyond the first-order kinetics law description was performed using the dispersive kinetics model developed by 


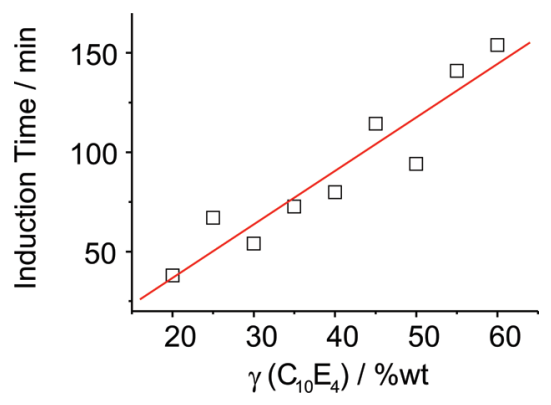

Figure 10. The length of the induction period preceding the phase transition from the ME phase to the LC phase exhibits a linear dependence on the surfactant concentration of the system.

Skrdla and Robertson. ${ }^{19}$ Dispersive kinetics are observed in systems that exhibit renewing conditions in which diffusion or mixing processes are rate limiting. The rate of molecular relaxation, the limited ability of a given reagent population to redistribute thermal energy, governs the overall conversion rate. A fundamental idea of this model is that molecular dynamic effects due to the different speeds and path lengths between collisions result in a dispersion in the activation energy. Instead of a single activation energy a convolution of the Maxwell-Boltzmann kinetic energy distribution and the traditional Arrhenius equation is used. The Maxwell-Boltzmann distribution, originally describing the distribution of speeds in an ideal gas at thermodynamic equilibrium, treats particles as distinguishable units which experience different energies due to their different spatial locations. A continuous depletion (via conversion) of the molecules in the high energy tail of the distribution leads to a subsequent redistribution of the remaining molecules in the bulk which reform this tail. In consequence, even at isothermal conditions, a varying number of molecules which are able to transform are present. This redistribution of thermal energy in a given chemical environment has a strong impact on the time scale of the conversion creating a time dependence in both the rate constant and the activation energy. The activation energy $E_{A}$ depends on the transformation time according to

$$
E_{\mathrm{A}}=E_{\mathrm{A}}^{0}+R T \beta t^{2}
$$

where $E_{\mathrm{A}}^{0}$ is a fixed potential energy barrier and $\beta$ a fit parameter that accounts for the time-dependent component of the activation energy. Here, $R$ is the gas constant, $T$ the absolute temperature, and $t$ the time. Microscopically, this distribution of activation energies leads to a distribution of molecular rate constants. As the transformation proceeds, the number of converting molecules changes like the corresponding rate constant. Macroscopically, a time-dependent rate constant $k(t)$ which is valid for one temperature can be observed for the whole transformation process

$$
k(t)=2 \alpha \beta \exp \left(-\beta t^{2}\right)
$$

In eq 7 and 9, the two fit parameters, $\alpha$ and $\beta$ with units of time ${ }^{-1}$ and time $^{-2}$, have a clear physical interpretation. $\alpha$ generally describes the induction period and the initial accumulation of product, while $\beta$ controls mainly the later stages of the conversion. $\alpha$ is defined as $\alpha=A^{n-1} \exp \left[-E_{\mathrm{A}}(T) / R T\right]$ where $E_{\mathrm{A}}$ is the time-independent activation energy of the phase transition, $A$ the Arrhenius frequency, and $n$ the dimension of the conversion. ${ }^{20} \beta$ is related to the activation entropy $\Delta S^{\ddagger}$ which

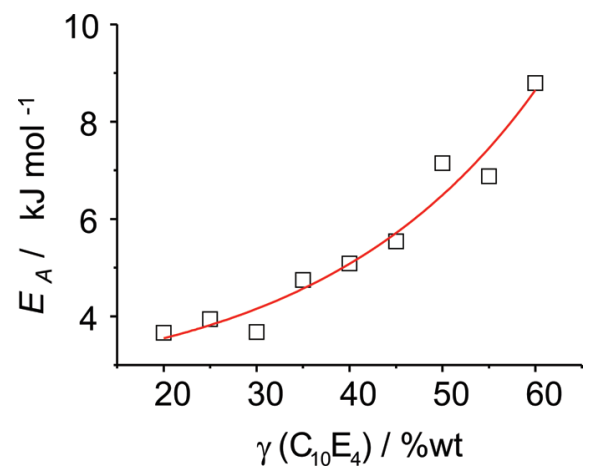

Figure 11. The activation energy for the ME-LC phase transition for various surfactant concentrations as determined according to the dispersive kinetics model. The values were extracted from regression fits of the experimental transformation profiles as displayed in Figure 9.

is time- and temperature- dependent

$$
\beta=\frac{\Delta S^{\dagger}}{R t^{2}}
$$

In dispersive kinetics, sigmoidal transformation curves which are often asymmetrical about their inflection points showing acceleratory or deceleratory behavior are frequently observed. These differences in shape were taken into consideration by the deviation of two cognate model equations. Skrdla et al. has shown that acceleratory conversion profiles, i.e., profiles which are characterized by a postinduction period in which the transformation speeds up are best described by

$$
I(t)=1-\exp \left\{[\alpha t]\left[\exp \left(-\beta t^{2}\right)-1\right]\right\}
$$

where $I(t)$ is the product fraction of the transformation. ${ }^{20}$ Deceleratory processes in contrast, conversions in which the rate constant for the transformation decreases as a function of the reaction time, are modeled by

$$
I(t)=1-\exp \left\{\left[-\alpha^{\prime} / t\right]\left[\exp \left(-\beta^{\prime} t^{2}\right)-1\right]\right\}
$$

The global kinetic parameters $\alpha^{\prime}$ and $\beta^{\prime}$, are used analogously to $\alpha$ and $\beta$ in eq 9 under isothermal conditions. In close relationship to the solid state, the formation of a LC can be thought of as a layer-by-layer process where the monomers are consecutively added at defect sides or grain boundaries. These structural similarities allow to apply the dispersive kinetics model to our observations of the investigated photoinduced phase transition. In Figure 9, the product fraction of the LC phase is expressed through the intensity increase $I(t)$ of is Bragg peak. It can be clearly seen that the regression fit of the dispersive kinetics model renders a good description of the experimental data for the phase transformation. As the surfactant concentration increases, smaller values of $\alpha$ which indicate increasing activation energies are determined. Hence, the extension of the induction time is a direct consequence of the change in the activation energy distribution. These findings are in turn explained by the concentration dependent properties of the initial ME. At small surfactant concentrations, there exist large oil and water domains. In contrast, in the case of high surfactant concentrations minor domains featuring small correlation lengths and $d$-spacing dominate the ensemble. The increased surface area of the small domains appears to increase simultaneously the activation energy for the phase transformation whereby the sharpness of this 
distribution is linked to the domain size of the ME. Smaller domains have a narrower distribution of energy states and a greater surface area resulting in higher overall surface energies than large domains. The opposite concentration dependence exists for the end state. The final LCs created at high surfactant concentrations are major sized consisting of a maximum number of lamellae. We recapitulate, that large LCs with many lamellae arise from a ME featuring a number of small domains. These antipodal proportions constitute a strong obstacle for the molecules, which needs to be overcome by diffusion processes. Within a lamellar phase only a reduced number of conformations are accessible and adoption of a preferred orientation of the molecules is required. This point coupled with the observation that the lamellae are most tightly packed at high surfactant concentrations accounts for the increase in activation energy which is demonstrated in Figure 11. Assigning a direct relation of the induction time and the activation energy of the ME-LC phase transformation according to Eyring leads to

$$
\begin{aligned}
\Delta G^{*}(T, t) & =E_{\mathrm{A}}^{0}(T)-2 R T-T \Delta S^{*}(T, t) \\
& =\Delta H^{*}(T)-T \Delta S^{*}(T, t)
\end{aligned}
$$

According to the classical transition state theory, here, $\Delta G^{\ddagger}$ is the free enthalpy of the transition state, $E_{\mathrm{A}}$ the activation energy, $\Delta H^{\ddagger}$ the transition state enthalpy, and $\Delta S^{\ddagger}$ the transition state entropy. It can be recognized that for an isoenergetical process with $\Delta H^{\ddagger}=$ constant, the activation energy $E_{\mathrm{A}}$ increases when the transformation entropy $\Delta S^{\ddagger}$ decreases. The entropy via

$$
\left(\frac{\partial S^{\sharp}}{\partial T}\right)_{p}=V
$$

is strongly connected with the temperature $T$ and the activation free volume $V$ around each monomer. Hence, the driving force of the ME/LC self-assembly is the gain in activation free volume during the self-assembly process. The large increase in translational entropy overcompensates losses in conformational and rotational entropy due to a reduced number of accessible states. ${ }^{21}$ This is also reflected in the increased surface area as plotted in Figure 7. Finally, the gain in activation free volume during the photoinduced phase transition leads to an increase of the transformation rate which expresses itself in the sigmoidal behavior and the found concentration dependence of the transformation kinetics.

\section{CONCLUSION}

In summary, we have demonstrated that the self-assembly process of amphiphilic molecules at the aqueous and LC interface can be induced by coherent optical radiation. Variation in the surfactant concentration reveals a major impact on the induction time and no significant effect on the period following the nucleation. The ordering scenario is determined by the diffusion process and the interaction of the dipole moment of the molecules with the electrical field producing a specific texture. The concentration dependence of the photoinduced phase transition results in an entropy driving process. After an ultrafast induction of the ME-LC phase transition, intrinsic dynamic features of the ternary mixture system take over and determine the rate constants of the process. The inherent thermal motion and the size of the ME domains vary in the chemical system as a function of surfactant concentration. This leads to changes in the distribution of activation energies that in turn defines a time dependent rate constant for the conversion and explains the origin of the concentration dependence of the induction time. Since the ME-LC phase transition is isoenergetic, the increase of free volume due to a tighter packing is caused by a decrease of rotational and translational degrees of freedom, decreasing the entropy contribution and allowing the phase transition to happen.

\section{ASSOCIATED CONTENT}

S Supporting Information. The conversion profiles as a function of irradiation time are given. As a first approximation, the conversion profiles are described by a first-order kinetic law (Figure 13) as well as, in an overlay, with the description according to the dispersive kinetics theory for sake of comparison. This material is available free of charge via the Internet at http://pubs.acs.org.

\section{AUTHOR INFORMATION}

\section{Corresponding Author}

*E-mail: stecher@gwdg.de. Phone: +49-551-201-1268. Fax: +49-551-201-1501.

\section{ACKNOWLEDGMENT}

This work was supported by DFG/SFB755 Nanoscale Photonic Imaging. Experiments were performed in part on the cSAXS beamline at the Swiss Light Source, Paul Scherrer Institut, Villigen, Switzerland.

\section{REFERENCES}

(1) Lockwood, N. A.; Gupta, J. K.; Abbott, N. L. Surf. Sci. Rep. 2008, $63,255$.

(2) Zhang, J. L.; Han, B. X.; Li, W.; Zhao, Y. J.; Hou, M. Q. Angew. Chem., Int. Ed. 2008, 47, 10119.

(3) Garetz, B. A.; Matic, J.; Myerson, A. S. Phys. Rev. Lett. 2002, 89.

(4) Delville, J. P.; Lalaude, C.; Buil, S.; Ducasse, A. Phys. Rev. E 1999, $59,5804$.

(5) Hallmann, J.; Morgenroth, W.; Paulmann, C.; Davaasambuu, J.; Kong, Q. Y.; Wulff, M.; Techert, S. J. Am. Chem. Soc. 2009, 131, 15018.

(6) Quevedo, W.; Petri, M.; Busse, G.; Techert, S. J. Chem. Phys. 2008, 129.

(7) Petri, M.; Busse, G.; Quevedo, W.; Techert, S. Materials 2009, 1305.

(8) Kahlweit, M.; Strey, R.; Haase, D.; Kunieda, H.; Schmeling, T.; Faulhaber, B.; Borkovec, M.; Eicke, H. F.; Busse, G.; Eggers, F.; Funck, T.; Richmann, H.; Magid, L.; Soderman, O.; Stilbs, P.; Winkler, J.; Dittrich, A.; Jahn, W. J. Colloid Interface Sci. 1987, 118, 436.

(9) (a) Sottmann, T.; Strey, R. J. Chem. Phys. 1997, 106, 8606. (b) Zhou, X. L.; Lee, L. T.; Chen, S. H.; Strey, R. Phys. Rev. A 1992, 46, 6479.

(10) Fournial, A. G.; Zhu, Y.; Molinier, V.; Vermeersch, G.; Aubry, J. M.; Azaroual, N. Langmuir 2007, 23, 11443.

(11) Quevedo, W.; Petri, M.; Techert, S. Z. Kristallogr. 2008, 223, 322.

(12) Teubner, M.; Strey, R. J. Chem. Phys. 1987, 87, 3195.

(13) Gunn, J. R.; Dawson, K. A. J. Chem. Phys. 1992, 96, 3152.

(14) Ciccariello, S.; Sobry, R. J. Appl. Crystallogr. 1999, 32, 892.

(15) Lichterfeld, F.; Schmeling, T.; Strey, R. J. Phys. Chem. 1986, 90, 5762.

(16) Atkin, R.; Warr, G. G. J. Phys. Chem. B 2007, 111, 9309. 
(17) Paz, L.; Di Meglio, J. M.; Dvolaitzky, M.; Ober, R.; Taupin, C. J. Phys. Chem. 1984, 88, 3415.

(18) Zhang, T. H.; Liu, X. Y. Angew. Chem., Int. Ed. 2009, 48, 1308.

(19) Skrdla, P. J.; Robertson, R. T. J. Phys. Chem. B 2005, 109, 10611.

(20) Skrdla, P. J. J. Phys. Chem. A 2009, 113, 9329.

(21) (a) Karayiannis, N. C.; Foteinopoulou, K.; Laso, M. Phys. Rev. Lett. 2009, 103. (b) Agmon, N. Biophys. J. 2005, 88, 2452. (c) Fournial, A. G.; Zhu, Y.; Molinier, V.; Vermeersch, G.; Aubry, J. M.; Azaroual, N. Langmuir 2007, 23, 11443. 